\title{
REMOVABLE SETS OF SUPPORT POINTS OF CONVEX SETS IN BANACH SPACES
}

\author{
R. R. PHELPS
}

\begin{abstract}
A corollary of the Bishop-Phelps theorem is that a closed convex subset $C$ of a Banach space can always be represented as the intersection of its supporting closed half-spaces. In this paper an investigation is made of those subsets $S$ of $C$ such that $C$ is the intersection of those closed half-spaces which support it at points of $C \backslash S$. This will be true for sets $S$ which are "small" relative to $C$, where smallness can be measured in terms of dimension, density character, or $\sigma$-compactness.
\end{abstract}

Suppose that $C$ is a nonempty closed convex subset of a Banach space $E$. A point $x \in C$ is called a support point of $C$ if there exists a nonzero functional $f \in E^{*}$ which attains its supremum on $C$ at $x$. Any such functional is said to be a support functional of $C$ and the set of all support points is denoted by supp $C$. It is known [1] that the support points of $C$ are always dense in bdry $C$, the boundary of $C$, and that the support functionals of $C$ are norm dense among those which are bounded above on $C$. A corollary of the methods used for these results is the fact that $C$ is always the intersection of all those closed half-spaces which are defined by support functionals [1, Corollary 2]. This result is trivial, of course, if $C$ has nonempty interior, since every boundary point of $C$ is a support point. In this case, in fact, it is easily seen that $C$ can be represented as the intersection of those halfspaces which support it at the points of $D$ for any dense subset $D$ of bdry $C$ (see part (iv) of Theorem 1, below). This fact has played a key role in characterizing those generators of $C_{0}$-semigroups of operators which leave invariant a given closed convex set with interior $[\mathbf{2}, \mathbf{3}]$. In considering the extension of his work [2] to more general convex sets, $\mathrm{K}$. N. Boyadzhiev raised the question (in a letter to the author) of whether one could express $C$ as the intersection of those closed half-spaces which support $C$ at some proper subset of supp $C$. The purpose of this note is to give some answers to this question.

A little thought shows that one has to use some care in deleting subsets of supp $C$. For instance, if $C$ is a line segment, then a point $x$ of $E \backslash C$ on the line determined by $C$ can be separated from $C$ only by those support functionals which attain their maximum on $C$ at the endpoint nearest $x$; that is, one cannot remove that endpoint from supp $C$ and still separate $x$ from $C$ by a support functional. (If the dimension of $E$ is at least two, then every point of $C$ is a support point, so $\operatorname{supp} C$ minus a single point is still dense in bdry $C$.) If $C$ is infinite dimensional, then one can remove a finite subset $S$ of supp $C$; in fact, as we show below, one can remove a finite dimensional subset and still obtain $C$ as the intersection of half-spaces which

Received by the editors October 7, 1985 and, in revised form, January 20, 1986.

1980 Mathematics Subject Classification (1985 Revision). Primary 46B20, 47D05.

Key words and phrases. Convex sets, Banach spaces, support points.

Work supported in part by a grant from the National Science Foundation. 
support it at the remaining points. The same conclusion will obtain if we remove a $\sigma$-compact set $S$ and $C$ is not $\sigma$-compact, or if $S$ is an infinite set whose density character is less than that of $C$. (The example of a segment described above shows why we have assumed that $S$ is infinite.) If the interior of $C$ is nonempty, then one can remove any subset of bdry $C$ whose complement is dense in the boundary.

Recall that the dimension $\operatorname{dim} A$ of a subset $A$ of a linear space is the algebraic dimension of its affine span. Recall, also, that the density character dens $A$ of a set $A$ is the smallest cardinal number $\alpha$ such that $A$ contains a dense subset of cardinality $\alpha$. We denote by $B_{r}(y)$ the open ball of radius $r>0$ centered at $y \in E$. If $\|x-y\|>r$, we define the drop $D(y, r, x)$ to be the convex hull of $\{x\}$ and the closed ball $B_{r}(y)^{-}$.

1. THEOREM. Suppose that $C$ is a nonempty closed convex subset of a Banach space $E$ and that $S$ is a subset of bdry $C$. Assume that either

(i) $S$ is finite dimensional and $1+\operatorname{dim} S<\operatorname{dim} C$, or

(ii) $S$ is infinite and dens $S<\operatorname{dens} C$, or

(iii) $S$ is relatively $\sigma$-compact and $C$ is not $\sigma$-compact, or

(iv) $C$ has nonempty interior and (bdry $C$ ) $\backslash S$ is dense in bdry $C$.

Then $C$ is the intersection of those closed half-spaces which support it at points of $C \backslash S$.

PROOF. Since the dimension of a finite-dimensional set (or the density character of any set) is the same as that of its closure, to prove the theorem under hypotheses (i), (ii), or (iii), we may assume without loss of generality that the set $S$ is closed. (We will prove part (iv) later.) Suppose that $y \in E \backslash C$, let $d=\operatorname{dist}(y, C$ ), and let $U=B_{5 d / 4}(y) \cap C$. This relatively open subset of $C$ is obviously nonempty; we will, in fact, show below that there exists a point $z \in U$ such that the segment $[z, y]$ misses $S$. Suppose that this has been done. Since $[z, y]$ misses the closed set $S$, some neighborhood of $[z, y]$ also misses $S$; in particular, for sufficiently small $0<\epsilon<d$ the drop $D(y, \epsilon, z)$ will miss $S$. From Brøndsted's proof (and subsequent remark) [3, Theorem 3] of Danes' "drop theorem" [4], there exists $x \in D(y, \epsilon, z)$ such that $D(y, \epsilon, x) \cap C=\{x\}$. The separation theorem applied to $C$ and the interior of $D(y, \epsilon, x)$ yields $f \in E^{*},\|f\|=1$, such that sup $f(C)=f(x)<f(y)$, that is, $f$ supports $C$ at a point $x$ of $C \backslash S$ and strictly separates $y$ from $C$.

It remains to show that under hypothesis (i), (ii), or (iii), we can choose $z \in U$ such that $[z, y]$ misses $S$. Suppose that this were not the case, that is, suppose that $[u, y]$ intersects $S$ for each $u \in U$. We will show first that this implies that the affine span $M$ of $C \cup\{y\}$ equals the affine span $L$ of $S \cup\{y\}$. Indeed, since $[u, y] \cap S \subset U$, we can assume without loss of generality that $0 \in S \cap U$ and take $L$ and $M$ to be the linear spans of $S \cup\{y\}$ and $C \cup\{y\}$, respectively. Obviously, $L \subset M$. Suppose that $x \in C$; then since 0 is a relative interior point of $C$, we can write $x=\lambda u$ for some $u \in U$ and $\lambda>0$. By hypothesis, there exists $0<\mu \leq 1$ such that $s \equiv \mu u+(1-\mu) y \in S$; a quick sketch will convince one that $x$ is in the linear span of $s$ and $y$, hence in $L$. [Explicitly, $x=\mu^{-1} \lambda\{s-(1-\mu) y\}$.] Since $C \subset L$ we must have $M=L$. This equality implies that

$$
1+\operatorname{dim} S \geq \operatorname{dim} \operatorname{sp}[S \cup\{y\}]=\operatorname{dim} \operatorname{sp}[C \cup\{y\}] \geq \operatorname{dim} C,
$$

which contradicts hypothesis (i). It also implies (if $S$ is an infinite set) that dens $S=$ dens $\operatorname{sp}[S \cup\{y\}]=\operatorname{dens} \operatorname{sp}[C \cup\{y\}]=\operatorname{dens} C$, which contradicts hypothesis (ii). 
Finally, if $S$ is $\sigma$-compact, then the subspace $L$ will be $\sigma$-compact; since $C \subset L$, we conclude that $C$ is $\sigma$-compact, contradicting hypothesis (iii).

Suppose, now, that the hypotheses in (iv) are satisfied. Let $D$ be the convex hull of $y$ and int $C$, the interior of $C$, and let $V=D \cap$ bdry $C$; this latter is obviously a nonempty relatively open subset of bdry $C$. Since (bdry $C$ ) \S is dense in bdry $C$, we can choose $z \in V \backslash S$. By the separation theorem there exists $g \in E^{*},\|g\|=1$, such that $\sup g(C)=g(z)$. Since $z \in V$, there exist $x \in$ int $C$ and $0<\lambda<1$ such that $z=\lambda x+(1-\lambda) y$. Necessarily, $g(x)<\sup g(C)$ and hence $g(y)>\sup g(C)$, that is, $g$ strictly separates $y$ from $C$, which completes the proof.

It is not difficult to show that if $C$ is infinite dimensional with nonempty interior, then any subset $S$ of bdry $C$ which is relatively locally compact or of first Baire category necessarily satisfies the density hypothesis in (iv).

While the conditions described in the theorem above are sufficient, none of them is necessary. For instance, if $C$ is a triangle in the plane, then it is the intersection of three half-spaces, so one can remove the entire boundary, with the exception of one point [resp. a proper open subinterval] in the relative interior of each side. This shows that the conclusion to the theorem can hold even when the hypotheses in either (i), (ii), or (iii) [resp. (iv)] fail. This example suggests a characterization of "removable" sets for finite-dimensional polyhedra pointed out to us by Thomas Armstrong: For such a polyhedron, the conclusion of Theorem 1 holds if and only if $C \backslash S$ intersects every maximal proper face of $C$. This result led Armstrong to formulate the following proposition, which gives a necessary condition for $S$ to be removable. We call a nonempty closed convex subset $F$ of a closed convex set $C$ with nonempty interior a face of $C$ provided there exists $g \in E^{*},\|g\|=1$, such that

$$
F=\{x \in C: g(x)=\sup g(C)\} .
$$

2. Proposition. Suppose that $E$ is finite dimensional and that $C$ is a bounded closed convex subset of $E$ having nonempty interior. If $S \subset C$ and if $C$ is the intersection of the closed half-spaces supported by (bdry $C) \backslash S$, then the closure $J$ of this latter set must intersect every maximal proper face $F$ of $C$.

Proof. Assume, without loss of generality, that $0 \in \operatorname{int} C$, and suppose that $F$ is a maximal proper face of $C$. Choose a point $y$ in the interior of $F$ (relative to the affine variety generated by $F$ ). Since $y$ is in bdry $C$, for any $\lambda>1$, the point $\lambda y$ is not in $C$. By hypothesis, then, for each $n \geq 1$ there exist $\left\|f_{n}\right\|=1$ and $x_{n} \in($ bdry $C) \backslash S$ such that $f_{n}\left[\left(1+n^{-1}\right) y\right]>\sup f_{n}(C)=f_{n}\left(x_{n}\right)$. Choose convergent subsequences (we call them $\left\{x_{n}\right\}$ and $\left\{f_{n}\right\}$ ) with $x_{n} \rightarrow x \in J$ and $f_{n} \rightarrow f$. If $w \in C$, then

$$
f(w)=\lim f_{n}(w) \leq \lim \inf f_{n}\left[\left(1+n^{-1}\right) y\right]=\lim f_{n}(y)=f(y),
$$

so $f(y)=\sup f(C)$. It is also easily shown that $f(x)=\sup f(C)$. Since $y$ is in the relative interior of $F$, we have $f(z)=\sup f(C)$ for every $z \in F$, that is, $F$ is a subset of the face $G=\{z \in C: f(z)=\sup f(C)\}$. The latter is proper since $f \neq 0$ and $C$ has nonempty interior. By maximality, this implies that $F=G$, and hence $x \in F \cap J$, which completes the proof.

It seems difficult to find a condition on $S$ which will be both necessary and sufficient for removability, even in the case when $C$ has nonempty interior. Our sufficient condition (iv) in Theorem 1 above requires that the set $J$ of Proposition 
2 be all of bdry $C$. Our next proposition shows that this can be sharpened by requiring that $J$ only intersect each maximal proper face of $C$, provided $C$ has nonempty interior and is smooth, that is, for each point $x$ in the boundary of $C$ there exists precisely one linear functional of norm one which attains its maximum on $C$ at $x$.

3. Proposition. Suppose that $C$ is a closed convex subset of $E$ which has nonempty interior and is smooth. If $S \subset C$ is such that the closure $J$ of (bdry $C$ ) \S intersects every maximal proper face of $C$, then $C$ is the intersection of those closed half-spaces which support it at the points of (bdry $C) \backslash S$.

Proof. Suppose that $y \in E \backslash C$. Let $U$ be the (relatively open) nonempty intersection of bdry $C$ and the convex hull $K$ of int $C \cup\{y\}$. Choose any point $x \in U$ and $f \in E^{*},\|f\|=1$, such that $f(x)=\sup f(C)$. Let $F$ be the closed face consisting of all points in $C$ where $f$ attains its maximum. It follows easily from the smoothness hypothesis that $F$ is maximal, hence $F \cap J$ is nonempty. Moreover, $F \subset U$; indeed, if there existed $z \in F \backslash U$, then necessarily $z \in$ bdry $K$ and we could choose $g \in E^{*},\|g\|=1$, such that $g(z)=\sup g(K) \geq \sup g(C)$. Since $g(z)=\sup g(C)=g(y)$ and $f(y)>\sup f(C)=f(z)$, we would have $f \neq g$, contradicting the smoothness hypothesis at the point $z$. Since $U$ is a relatively open neighborhood of $F$, the fact that $F$ intersects $J$ implies that there is a point of (bdry $C) \backslash S$ in $U$. The support functional to $C$ at such a point will strictly separate $y$ from $C$ (as shown at the end of the proof of Theorem 1).

If $C$ has nonempty interior and is strictly convex, then to say that $J$ intersects every maximal proper face is equivalent to saying that (bdry $C) \backslash S$ is dense in bdry $C$. Thus, for strictly convex and smooth $C$ with interior, Proposition 3 is no more general than Theorem 1 (iv).

ACKNOWLEDGMENT. We wish to express our gratitude to Professor Thomas Armstrong, who, during several conversations at the 1985 International Conference on Banach Spaces and Classical Analysis at Kent State University, conjectured Propositions 2 and 3 and made the observation about finite-dimensional polytopes which precedes them.

\section{REFERENCES}

1. Errett Bishop and R. R. Phelps, The support functionals of a convex set, Proc. Sympos. Pure Math., vol. 7, Amer. Math. Soc., Providence, R. I., 1963, pp. 27-35.

2. K. N. Boyadzhiev, Characterization of the generators of $C_{0}$ semigroups which leave a convex set invariant, Comment. Math. Univ. Carolinae 25 (1984), 159-170.

3. A. Brøndsted, On a lemma of Bishop and Phelps, Pacific J. Math. 55 (1974), 335-341.

4. J. Danes, A geometric theorem useful in nonlinear functional analysis, Boll. Un. Mat. Ital. (4) 6 (1972), 369-375.

5. R. Martin, Invariant sets for evolution systems, International Conf. on Diff. Equations, Academic Press, New York, 1975.

Department of Mathematics GN-50, University of Washington, Seattle, WASHINGTON 98195 